Volume: 2, Issue: 1, January-March 2017, Pages: 146, DOI: http://dx.doi.org/10.19082/ah146

\title{
ASSESSING SMARTPHONE APPLICATIONS FOR HYPERTENSION MANAGEMENT
}

\author{
F Rangrazejeddi ${ }^{1}$, RS Sharif ${ }^{2}$, Sh Anvari ${ }^{3}$
}

1: Associate Professor, Health Information Management Research Center, Kashan University of Medical Sciences, Kashan, Iran.

2: MS student, Health Information Management Research Center, Kashan University of Medical Sciences, Kashan, Iran.

3: MS student, Health Information Management Research Center, Kashan University of Medical Sciences, Kashan, Iran.

Correspondence:

RS Sharif, E-mail: Fr.sharif@yahoo.com, Tel: +98-914-0457394.

\section{TYPE OF ARTICLE: CONFERENCE ABSTRACT}

\begin{abstract}
Introduction: Hypertension is a disease that can lead to heart failure, heart attack, and stroke. Therefore, it is essential for patients to have self-management skills. Smartphone-based medical applications (apps) can facilitate self-management of hypertension (HTN). Several applications have been provided for these patients, but the content and quality of these applications have not been evaluated. The aim of this study was to assess the quality of free applications available on the Android platform using a set of content-independent quality criteria developed by the Health on the Net Foundation for Hypertension in Iran.

Methods: This unsystematic study was conducted by searching in BAZAR Market (an Android market of applications for smartphones in Iran) in 2016, using the search terms hypertension, high blood pressure, control and treatment of hypertension. Each HTN app included in the study was coded into eight possible HON (Health on the Net Foundation) codes: (1) information must be authoritative; (2) purpose of the app; (3) confidential; (4) information must be documented, referenced and dated; (5) justification of claims; (6) application contact details; (7) funding; and (8) editorial and advertising policy. Each application was coded by two independent researchers. Each application received a " 0 " or " 1 ," a " 0 " for not meeting the HON code requirement, and 1 for meeting the HON code requirement.

Results: According to the searches, 25 free applications related to hypertension. The range of health information quality for all the HTN applications was between 0 and 2. In all, only nine out of the 25 HTN applications listed contact details. None of the applications clearly indicated how the application was funded. None of the applications did meet the following criteria: Reliability of information, the purpose of the application, based on scientific evidence, confidentiality, update information, funding and editorial, and advertising policy.

Conclusion: This study shows that quality of Persian applications relating to hypertension in all criteria is poor. It is essential that developers and designers in addition to improving and solving weaknesses seek to create applications based on scientific evidence. In this regard, more interaction with professional health care organizations and academic centers is recommended.
\end{abstract}

KEYWORDS: Hypertension, Mobile health, Application, Android

\footnotetext{
Abstracts of First National Congress of Medical Informatics, Mashhad, Iran, February 2017

(C) 2017 The Authors. This is an open access article under the terms of the Creative Commons Attribution-NonCommercialNoDerivs License, which permits use and distribution in any medium, provided the original work is properly cited, the use is non-commercial and no modifications or adaptations are made.
} 